\title{
The relativity of color perception
}

\author{
Michel Berthier*1, Valérie Garcin ${ }^{\dagger 2}$, Nicoletta Prencipe ${ }^{\ddagger 3}$ and Edoardo Provenzi ${ }^{\S 4}$ \\ ${ }^{1}$ Laboratoire MIA, Pôle Sciences et Technologie, Université de La Rochelle, \\ Avenue Michel Crépeau, 17042 La Rochelle Cedex 1 \\ ${ }^{2,3,4}$ Université de Bordeaux, CNRS, Bordeaux INP, IMB, UMR 5251, F-33400, 351 \\ Cours de la Libération, Talence, France
}

\begin{abstract}
The physiological mechanisms of the neural coding of colors aim at explaining how physical colors, i.e. reflected or emitted light entering the eyes from a visual environment, are converted into perceived colors, i.e. the colors that can be sensed by humans. These mechanisms are well-known to involve both three separate receptor types, the LMS cones, and spectrally opponent and non-opponent interactions resulting from the activity rates of ganglion and lateral geniculate nucleus cells. Color perception is thus a process inherently linked to an observing apparatus and to an experimental environment. The main objective of this contribution is to present a rigorous mathematical model that allows, by taking into account both trichromacy and color opponency viewpoints, to explain relativistic color perception phenomena first argued by Yilmaz in 1962. The cornerstone of the proposed approach is the description of Hering's opponency by means of a quantum system, namely a rebit, whose (chromatic) states are shown to satisfy Einstein-Poincaré relativistic addition law. This implies for instance that the Hilbert metric on the state space of the rebit is relevant to express a chromatic constancy property with respect to observer changes, as confirmed by measurements on experimental data.
\end{abstract}

\section{Introduction}

As already pointed out by Russell 22, color perception is a phenomenon that cannot be investigated without making reference to an observing apparatus and to an experimental environment: "When, in ordinary life, we speak of the colour of the table, we only mean the sort of colour which it will seem to have to a normal spectator from an ordinary point of view under usual conditions of light. But the other colours which appear under other conditions have just as good a right to be considered real; and therefore, to avoid favoritism, we are compelled to deny that, in itself, the table has any one particular colour". This point of view is clearly much more reminiscent of the way one addresses the problem of measurements in quantum mechanics rather than in classical mechanics.

All these observations seem to indicate that a quantum theory of color perception, whose language is developed in section 2, is more adequate than a classical one. As we will discuss in section 3 this intuition, already guessed in [1] and strongly supported by the mathematical results obtained in 20, has been rigorously formalized in 3 .

Recollecting results from [3] and [19, we explain how to obtain a meaningful geometrical structure on the space of perceived colors by using only the so-called trichromacy axiom, which

\footnotetext{
*michel.berthier@univ-lr.fr

†valerie.garcin@math.u-bordeaux.fr

${ }_{\ddagger}^{\ddagger}$ nicoletta.prencipe@math.u-bordeaux.fr

§edoardo.provenzi@math.u-bordeaux.fr
} 
can be seen as an extended version of Schrödinger and Resnikoff's set of axioms. As suggested by its name, this axiom is meant to sum up in a mathematical language what is known concerning the trichromatic aspects of color perception. Surprisingly, it can be shown that this sole axiom is sufficient to bring out, from the properties of Jordan algebras, a quantum dynamic that fully models Hering's opponency mechanisms. This allows us to provide a coherent quantum framework which we will also prove to be compatible with a relativistic theory of color perception.

Section 4 is devoted to the description of Yilmaz contribution [26. Inspired by the mathematical physics of the special theory of relativity, the author explained how to derive colorimetric analogues of Lorentz transformations by exploiting the results of three color matching experiments. Yilmaz experiments are precisely described and analyzed in 18, and they are recalled in the appendix at the end of our paper. Our aim in this section is to follow Yilmaz as close as possible. Nevertheless, we adapt the presentation and the argumentation in order to emphasize what information is really taken into account and how it can be used from the mathematical viewpoint. This motivates the introduction of a new nomenclature in section 4.2 with, in particular, the precise definition of an observer adapted to an illuminant in the context considered by Yilmaz.

As discussed in section 4.5, the outcome of Yilmaz experiments is quite controversial, for this reason, starting from section 5 , we prove that color perception is a relativistic phenomenon bypassing Yilmaz experiments and by using only the trichromacy axiom. The main source of inspiration that guided us during this task is represented by the remarkable Mermin's paper [16], in which it is shown that the core aspect of special relativity is better understood if one concentrates not on Lorentz transformations but on Einstein-Poincaré addition law of velocity vectors. This will lead us directly to the definition, in section 5.1. of the concept of perceptual chromatic vector, alongside with a whole new additional set of definitions regarding quantum perceptual chromatic attributes.

The purely theoretical proof that perceptual chromatic vectors satisfy the Einstein-Poincaré addition law, performed in section 5.2, not only permits to recast the outcome of the first two Yilmaz experiments in our mathematically rigorous framework, but is also confirms, see section 6. the relevance of the Hilbert metric, first introduced in the context of color perception in $\underline{3}$, on the space of perceptual chromatic vectors: in this context, the Hilbert distance expresses a chromatic constancy property with respect to observer changes. In section 6.1 we show that our theoretical results are coherent with experimental data.

Finally, in section 7, we explain how to theoretically recover the result given by the third experiment of Yilmaz, which is crucial in his approach since it avoids having to resort to a hypothetical perceptual invariant Minkowski-like quadratic form. Yilmaz already noticed that the corresponding chromatic effect can be considered as an analogue of relativistic aberration. To recover this effect, we essentially show that pure perceptual chromatic states generate one-parameter subgroups of Lorentz boost maps. This is a key result that links the quantum dynamics of chromatic opponency with the relativity of color perception.

\section{The nomenclature of quantum perception}

As stated in the introduction, we will deal with a quantum theory of color perception. This implies the need of a suitable nomenclature that we establish in this section and that is adapted from the language of quantum physics. From [7, 24, 17, we recall that the axiomatic structure of classical and quantum theories is very similar, the fundamental difference being represented by the so-called algebra of observables of the theory, as defined as follows:

- a physical system $\mathcal{S}$ is described as a setting where one can perform physical measures giving rise to quantitative results in conditions that are as isolated as possible from external influences;

- observables in $\mathcal{S}$ are the objects of measures. If they form an associative and commutative algebraic structure, then the physical theory is called classical, if either one or both properties lack, then we talk about a quantum theory;

- states of $\mathcal{S}$ are associated with the ways $\mathcal{S}$ is prepared for the measurement of its observables; 
- the expected value of an observable in a given state of $\mathcal{S}$ is the average result of multiple measures of the observable conducted in the physical system $\mathcal{S}$ prepared in the same state.

Regarding this last definition, we notice that this is the standard experimental way of associating a value to an observable both in classical and in quantum physics for two different reasons: in the former we assume that nature is deterministic and we need to introduce the expected value because all measurements are affected by errors, instead in the latter we assume that nature is intrinsically probabilistic and so we need the concept of expected value to single out a value from the set of values that the observable can assume.

Observables characterize a state through their measurements and, vice-versa, the preparation of a particular state characterizes the experimental results that will be obtained on the observables. In the standard Hilbert space formulation of quantum theories, observables are Hermitian operators on a Hilbert space, thus they form an associative but non-commutative algebra and the duality observable-state is mathematically formalized by the Riesz-Markov-Kakutani representation theorem 21. However, as we will see next, in the alternative quantum description proposed by Jordan in [12], observables are elements of a commutative but non-associative Jordan algebra

and the duality observable-state in this case can be encoded in the autoduality property of the positive cone of this algebra.

When we deal with a perceptual system, as e.g. a light stimulus embedded in a homogeneous background, the definitions above remain valid, with two major differences: first, the instruments used to measure the observables are not physical devices, but the sensory system of a human being; second, the results may vary from person to person, thus the average procedure needed to experimentally define the expected value of an observable in a given state is, in general, observerdependent. The response of an ideal standard observer can be obtained through a further statistical average on the observer-dependent expected values of an observable in a given state.

If we specialize this idea to the case of color perception, we may give the following colorimetric definitions:

- a perceptual chromatic state is represented by the preparation of a visual scene for psychovisual experiments;

- a perceptual color is the perceptual observable identified with a psycho-visual measure performed in a given perceptual chromatic state;

- a perceived color is the expected value assumed by a perceptual color after a psycho-visual measure.

We underline that the definition of a perceptual color as an observable associated to a psychovisual measure in a given perceptual chromatic state is very different than the physical meaning of the term 'color stimulus', i.e. the spectral distribution of a light signal across the visual interval. In fact, such a color stimulus, presented to an observer in different conditions, e.g. isolated or in context, can be sensed as dramatically different perceived colors. Thus, from a perceptual viewpoint, it is very ill-posed to identify a perceptual color with a color stimulus, as also mentioned in [25], a classical reference for colorimetry.

\section{Trichromacy and quantum color opponency}

In this section we present the quantum theory of color perception on which the rest of the paper will be based upon. We present just an overview of the results obtained in [3] and [19], we refer the reader to these papers for details and explanations, especially in what concerns Jordan algebras.

\subsection{Color perception from the trichromacy axiom}

The path that leads to the so-called trichromacy axiom can be succinctly summarized as follows. The classical, and well established, colorimetric experiences of Newton, Grassmann and Helmholtz have been resumed by Schrödinger in a set of axioms that describe the structure of a space designed to represent the set of colors from the trichromatic properties of color perception. These axioms stipulate that this space, denoted $\mathcal{C}$ from now on, is a regular convex cone of real dimension 3 . It 
is very important to note that, although it seems tempting to consider $\mathcal{C}$ as the space of perceived colors, it is much more appropriate to consider it as a space of perceptual observables as defined above. To avoid confusion, we call $\mathcal{C}$ the trichromacy cone. In [7], Resnikoff showed that to fully exploit this mathematical structure one needs to add a supplementary axiom, namely the fact that $\mathcal{C}$ is homogeneous, which precisely means that there exists a group acting transitively on $\mathcal{C}$. If we add one more property, the self-duality of $\mathcal{C}$, then $\mathcal{C}$ becomes a symmetric cone. According to the Koecher-Vinberg theorem 2], the trichromacy $\mathcal{C}$ can then be seen as the positive cone of a formally real Jordan algebra $\mathcal{A}$, i.e. as the set of squares of $\mathcal{A}$. This motivates the following:

Trichromacy AXIOM [3]: - The trichromacy cone $\mathcal{C}$ is the positive cone of a formally real Jordan algebra of real dimension 3.

The idea to recast the study of color perception in the Jordan algebra framework appears already in Resnikoff's contribution [20. However, Resnikoff was more interested by using this concept to understand brightness than to give a quantum interpretation of the classical axioms of Schrödinger. Note that our trichromacy axiom differs from these latter by the fact that we require $\mathcal{C}$ to be homogeneous and self-dual. Self-duality implies that $\mathcal{C}$ can also be considered as the state cone associated to the perceptual chromatic state space. As mentioned before, this formulation of the trichromacy axiom aims at emphasizing the observable-state duality on which our approach relies.

The most surprising and intriguing consequences of the trichromacy axiom are given by the classification theorem of Jordan - von Neumann - Wigner, see for instance [2]. According to this theorem, the Jordan algebra $\mathcal{A}$ is isomorphic either to the sum $\mathbb{R} \oplus \mathbb{R} \oplus \mathbb{R}$ or to the Jordan algebra $\mathcal{H}(2, \mathbb{R})$ of 2 by 2 symmetric matrices with real entries. The positive cone of the sum $\mathbb{R} \oplus \mathbb{R} \oplus \mathbb{R}$ is the product $\mathbb{R}^{+} \times \mathbb{R}^{+} \times \mathbb{R}^{+}$. When equipped with the so-called Helmholtz-Stiles metric:

$$
d s^{2}=\sum_{i=1}^{3} a_{i}\left(d \xi_{i} / \xi_{i}\right)^{2},
$$

$a_{i}, \xi_{i} \in \mathbb{R}^{+}$, it is the metric space used in standard colorimetry. Since this space has been extensively studied, in the sequel we will concentrate only on the second possibility which, as we will see, contains the quantum structure that we are looking for. A crucial remark is that $\mathcal{H}(2, \mathbb{R})$ is isomorphic, as a Jordan algebra, to the spin factor $\mathbb{R} \oplus \mathbb{R}^{2}$ whose Jordan product is defined by:

$$
\left(\alpha_{1}, \mathbf{v}_{1}\right) \circ\left(\alpha_{2}, \mathbf{v}_{2}\right)=\left(\alpha_{1} \alpha_{2}+\left\langle\mathbf{v}_{1}, \mathbf{v}_{2}\right\rangle, \alpha_{1} \mathbf{v}_{2}+\alpha_{2} \mathbf{v}_{1}\right)
$$

where $\alpha_{1}$ and $\alpha_{2}$ are reals, $\mathbf{v}_{1}$ and $\mathbf{v}_{2}$ are vectors of $\mathbb{R}^{2}$ and $\langle$,$\rangle denotes the Euclidean scalar$ product on $\mathbb{R}^{2}$. An explicit isomorphism of Jordan algebras is given by:

$$
\chi:(\alpha, \mathbf{v}) \in \mathbb{R} \oplus \mathbb{R}^{2} \longmapsto\left(\begin{array}{cc}
\alpha+v_{1} & v_{2} \\
v_{2} & \alpha-v_{1}
\end{array}\right) \in \mathcal{H}(2, \mathbb{R}),
$$

where $\mathbf{v}=\left(v_{1}, v_{2}\right)$.

The positive cone of the Jordan algebra $\mathcal{H}(2, \mathbb{R})$ is the set of positive semi-definite 2 by 2 symmetric matrices with real entries. Via the isomorphism $\chi$ it corresponds to the positive cone of the spin factor $\mathbb{R} \oplus \mathbb{R}^{2}$. This latter is given by:

$$
\mathcal{C}=\left\{(\alpha, \mathbf{v}) \in \mathbb{R} \oplus \mathbb{R}^{2}, \alpha^{2}-\|\mathbf{v}\|^{2} \geq 0, \alpha \geq 0\right\} .
$$

The cone $\mathcal{C}$ is the trichromacy cone.

\subsection{Quantum color opponency}

$\mathcal{H}(2, \mathbb{R})$ is the algebra of observables of the real analogue of a qubit called a rebit. The states of this quantum system are characterized by density matrices, i.e. positive elements of $\mathcal{H}(2, \mathbb{R})$ with trace equal to 1 . It is clear that a matrix of $\mathcal{H}(2, \mathbb{R})$ written as in eq. (3) is a density matrix if 
and only if $\mathbf{v}=\left(v_{1}, v_{2}\right) \in \mathcal{D}=\left\{v \in \mathbb{R}^{2},\|v\| \leq 1\right\}$ and $\alpha=1 / 2$. Two explicit expressions for the density matrices are:

$$
\rho(\mathbf{v})=\frac{1}{2}\left(\begin{array}{cc}
1+v_{1} & v_{2} \\
v_{2} & 1-v_{1}
\end{array}\right),
$$

and

$$
\rho(\mathbf{v})=\frac{1}{2}\left(I d_{2}+\mathbf{v} \cdot \sigma\right)=\frac{1}{2}\left(I d_{2}+v_{1} \sigma_{1}+v_{2} \sigma_{2}\right),
$$

where $I d_{2}$ is the $2 \times 2$ identity matrix and $\sigma=\left(\sigma_{1}, \sigma_{2}\right)$, with:

$$
\sigma_{1}=\left(\begin{array}{cc}
1 & 0 \\
0 & -1
\end{array}\right) \quad \sigma_{2}=\left(\begin{array}{ll}
0 & 1 \\
1 & 0
\end{array}\right) .
$$

If we consider the state vectors written in Dirac's notation as the following set of 'ket':

$$
\left|u_{1}\right\rangle=\left(\begin{array}{l}
1 \\
0
\end{array}\right) \quad\left|d_{1}\right\rangle=\left(\begin{array}{l}
0 \\
1
\end{array}\right) \quad\left|u_{2}\right\rangle=\frac{1}{\sqrt{2}}\left(\begin{array}{l}
1 \\
1
\end{array}\right) \quad\left|d_{2}\right\rangle=\frac{1}{\sqrt{2}}\left(\begin{array}{c}
-1 \\
1
\end{array}\right),
$$

then, by denoting with $\langle|=|\rangle^{t}$ the corresponding set of 'bra', we have:

$$
\sigma_{1}=\left|u_{1}\right\rangle\left\langle u_{1}|-| d_{1}\right\rangle\left\langle d_{1}\right| \text { and } \sigma_{2}=\left|u_{2}\right\rangle\left\langle u_{2}|-| d_{2}\right\rangle\left\langle d_{2}\right| .
$$

This shows that the state vectors $\left|u_{1}\right\rangle$ and $\left|d_{1}\right\rangle$, resp. $\left|u_{2}\right\rangle$ and $\left|d_{2}\right\rangle$, are eigenstates of $\sigma_{1}$, resp. $\sigma_{2}$, with eigenvalues 1 and -1 .

The two matrices $\sigma_{1}$ and $\sigma_{2}$ are Pauli-like matrices that give a two direction opponency mechanism. More precisely, representing $\left(v_{1}, v_{2}\right)$ in polar coordinates $(r, \theta)$, with $r \in[0,1]$ and $\theta \in[0,2 \pi)$, the density matrix $\rho(\mathbf{v})$ can be written in three equivalent forms:

$$
\rho(r, \theta)=\frac{1}{2}\left(\begin{array}{cc}
1+r \cos \theta & r \sin \theta \\
r \sin \theta & 1-r \cos \theta
\end{array}\right)
$$

or:

$$
\begin{gathered}
\rho(r, \theta)=\frac{1}{2}\left((1+r \cos \theta)\left|u_{1}\right\rangle\left\langle u_{1}|+(1-r \cos \theta)| d_{1}\right\rangle\left\langle d_{1}\right|\right. \\
\left.+(r \sin \theta)\left|u_{2}\right\rangle\left\langle u_{2}|-(r \sin \theta)| d_{2}\right\rangle\left\langle d_{2}\right|\right),
\end{gathered}
$$

or, by noticing that $\sigma_{1}=\rho(1,0)-\rho(1, \pi)$ and $\sigma_{2}=\rho(1, \pi / 2)-\rho(1,3 \pi / 2)$ and using eq. (6):

$$
\rho(r, \theta)=\rho_{0}+\frac{r \cos \theta}{2}(\rho(1,0)-\rho(1, \pi))+\frac{r \sin \theta}{2}(\rho(1, \pi / 2)-\rho(1,3 \pi / 2)),
$$

where $\rho_{0}=I d_{2} / 2$ and $\rho(r, \theta)=\rho_{0}$ if and only if $r=0$. A useful representation of the rebit states is provided by the Bloch disk, see Figure 1 .

The density matrices parameterized by $r=1$, independently on $\theta \in[0,2 \pi)$, i.e. $\rho(1, \theta)$, correspond to pure states. They are characterized by either the equation:

$$
\rho(1, \theta) \circ \rho(1, \theta)=\rho(1, \theta),
$$

or, recalling that the von Neumann entropy of a generic density matrix $\rho$ is defined as - Trace $(\rho \log \rho)$, by:

$$
\text { - } \operatorname{Trace}(\rho(1, \theta) \log \rho(1, \theta))=0
$$

which means that their von Neumann entropy is zero. These pure state density matrices are the projector matrices:

$$
\rho(1, \theta)=|1, \theta\rangle\langle 1, \theta|,
$$

with $|1, \theta\rangle=\cos (\theta / 2)\left|u_{1}\right\rangle+\sin (\theta / 2)\left|d_{1}\right\rangle$. The density matrix $\rho_{0}$ that corresponds to the state of maximal von Neumann entropy is $\rho_{0}=I d_{2} / 2$ and it can be written as the mixture:

$$
\rho_{0}=\frac{1}{4} \rho(1,0)+\frac{1}{4} \rho(1, \pi)+\frac{1}{4} \rho(1, \pi / 2)+\frac{1}{4} \rho(1,3 \pi / 2) .
$$




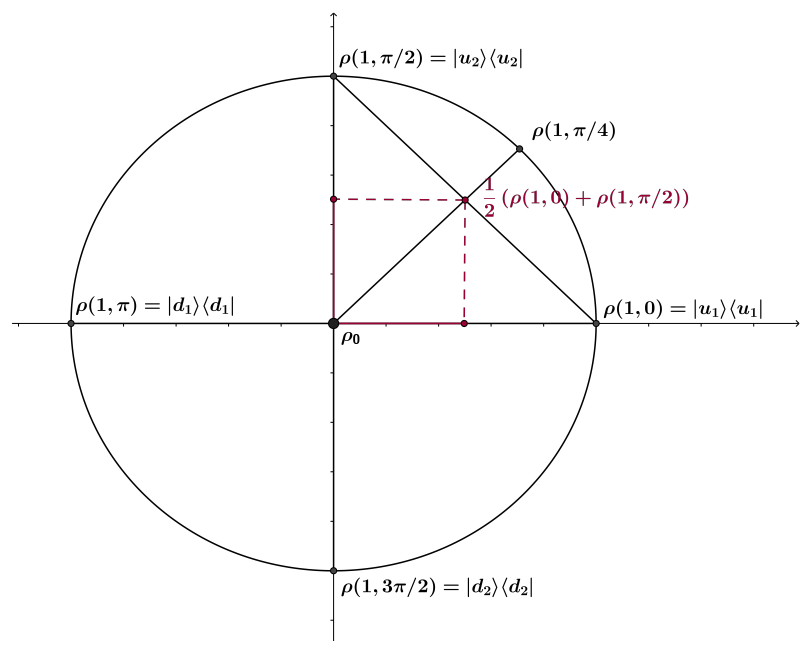

Figure 1: The Bloch disk of the rebit illustrating the opponency mechanism. The density matrix $\rho(1, \pi / 4)$ is given by: $\rho(1, \pi / 4)=|1, \pi / 4\rangle\left\langle 1, \pi / 4\left|=\left(\left|u_{1}\right\rangle+\left|u_{2}\right\rangle\right)\left(\left\langle u_{1}\right|+\left\langle u_{2}\right|\right) /(2+\sqrt{2})\right.\right.$. The mixture $(\rho(1,0)+\rho(1, \pi / 2)) / 2$ is the density matrix: $\rho(\sqrt{2} / 2, \pi / 4)=\rho_{0}+\frac{1}{4}(\rho(1,0)-\rho(1, \pi))+$ $\frac{1}{4}(\rho(1, \pi / 2)-\rho(1,3 \pi / 2))$.

Eq. (12) shows that every density matrix is the sum of the state of maximal entropy with two other components that describe the opponency with respect to the two directions $(\rho(1,0), \rho(1, \pi))$ and $(\rho(1, \pi / 2), \rho(1,3 \pi / 2))$. Given a density matrix $\rho(r, \theta)$, one can evaluate the contribution of the opposition $(\rho(1,0), \rho(1, \pi))$ given by $\sigma_{1}$ by computing:

$$
\left\langle\sigma_{1}\right\rangle_{\rho(r, \theta)}=\operatorname{Trace}\left(\rho(r, \theta) \circ \sigma_{1}\right)=r \cos \theta,
$$

and the same for the other direction. It is quite remarkable that the Block disk of Figure 1 gives a quantum analogue of the Hering disk that describes the color opponency mechanism resulting from the activity of certain retinal neurons $\left[23\right.$. The matrix $\sigma_{1}$ encodes the opposition red/green, while the matrix $\sigma_{2}$ encodes the opposition yellow/blue. We underline that this quantum justification of the color opponency derives only from the trichromacy axiom when considering the algebra $\mathcal{H}(2, \mathbb{R})$.

\section{Yilmaz relativity of color perception}

As we have declared in the introduction, one of the aims of our paper is to show that the quantum framework previously described is also perfectly suited to describe a relativistic theory of color perception, as we will show starting from section 5

Before going through the details of our analysis, it is necessary to discuss Yilmaz's paper [26] which, to the best of our knowledge, is the first and only contribution that investigates the geometry of color perception from the viewpoint of special relativity. The main Yilmaz goal is to obtain colorimetric Lorentz transformations by interpreting mathematically the outcomes of three basic experiments. Actually, as we will detail in section 4.5, these experiments are quite controversial and this fact gives an even stronger motivation to recast Yilmaz in a rigorous mathematical setting where these experiments can be completely bypassed. 


\subsection{Yilmaz colorimetric setting}

In order to analyze the results of color matching experiments, Yilmaz considers a conical color space that, in our notation, can be written as follows:

$$
\widetilde{\mathcal{C}}=\left\{(\alpha, x, y) \in \mathbb{R}^{3}, \Sigma^{2}-\|\mathbf{v}\|^{2} \geq 0, \alpha \geq 0\right\},
$$

where $\mathbf{v}=\left(v_{1}, v_{2}\right)=(x / \alpha, y / \alpha)$ with $\alpha>0$; if $\alpha=0$, then also $\mathbf{v}$ is null. It should be noticed that the cone $\widetilde{\mathcal{C}}$ used here is very similar to the trichromacy cone $\mathcal{C}$ introduced in section 3.1. But, contrary to our approach that leads naturally to consider this cone from the trichromacy axiom, the mathematical arguments, partially based on Fourier analysis, developed in 26] to justify the relevance of this space are not fully mathematically convincing. Moreover, as explained before, the cone $\mathcal{C}$ inherits of a rich algebraic structure which is not the case for $\widetilde{\mathcal{C}}$. A color $c$ of $\widetilde{\mathcal{C}}$ can be viewed both as a point of $\mathbb{R}^{3}$ with coordinates $(\alpha, x, y)$ and as a couple $(\alpha, \mathbf{v})$, where $\alpha$ is a positive real number and $\mathbf{v}$ is a vector of $\mathbb{R}^{2}$ of Euclidean norm $v=\|\mathbf{v}\|$ less or equal to $\Sigma$. In Yilmaz context, the norm $v$ is the saturation of the color $c$, the angle defined by $\phi=\arctan (y / x)$ its hue, and the positive real $\alpha$ its lightness.

The existence of a positive real $\Sigma$, which plays the role of a limiting saturation 'reached by spectral colors', is one of the fundamental assumptions of Yilmaz. The definition of saturation given above is the analogue of speed (the magnitude of the velocity vector) in mechanics, thus it seems clear that, from Yilmaz's viewpoint, the limiting saturation $\Sigma$ should be interpreted as an analogue of the light speed. Concisely, the purpose of the three experiments described in [26] is to show that:

1. color perception is a relativistic phenomenon;

2. the limiting saturation is constant under 'illuminant changes';

3. there exists a colorimetric aberration effect which is the analogue of the relativistic one.

It is worth mentioning that Yilmaz does not use any information related to a hypothetical invariant quadratic form. In physics, the introduction of an invariant metric on the Minkowski space is motivated by an abundance of precisely experimental facts, which are not available in the colorimetric setting. It is arguable that this is the reason why Yilmaz wanted to bypass the introduction of an invariant metric by introducing the results of the third experiment.

Similarly to what we have done in section 2 by adapting quantum mechanical definitions to the perceptual domain, our description and subsequent analysis of Yilmaz's experimental results will be greatly simplified if we set up a novel nomenclature, this time adapted from special relativity.

\subsection{The nomenclature of color perception relativity}

We start by underling that the perceptual chromatic states considered by Yilmaz are prepared in such a way that no emitting sources of light are present in the visual scene, for this reason, the existence of an illuminant is necessary to define the state.

Without any further specification, we consider a color $c$ as an abstract coordinate-free element of the space $\widetilde{\mathcal{C}}$. This interpretation is the exact analogue to what we do in Galilean mechanics when we consider the position as an abstract element of the space $\mathbb{R}^{3}$ without coordinates. A coordinate system can be introduced in $\widetilde{\mathcal{C}}$ by considering an illuminant ${ }^{1}$ which allows us to identify $c$ and to perform measurements on it. For this reason, here we define an illuminant to be a reference frame I of the space $\widetilde{\mathcal{C}}$.

It is well-known, see e.g. 8, 10, that when an observer is embedded for a sufficient time in a visual scene illuminated by $I$, he/she will perceive the surface of an object characterized by non-selective reflectance properties without a color saturation. In this case, we call that observer adapted to $I$. This consideration naturally leads us to call any couple $o=(c, I)$, such that the color $c \in \widetilde{\mathcal{C}}$ has zero saturation in the reference frame $I$, an observer adapted to an illuminant $I$,

\footnotetext{
${ }^{1}$ More precisely, we should call it a broadband illuminant, i.e. a light source extended over the entire visible spectrum. The reason is that, if we consider a narrow-band illuminant, the so-called Helson-Judd effect enters into play and an observer will experience an incomplete adaptation, see e.g. [8]. For the sake of simplicity, we will implicitly consider an illuminant as broadband without further specifications.
} 
or simply an observer from now on. Given the analogy between the saturation of a color and the speed of a velocity vector for a mechanical system, an observer $o=(c, I)$ is characterized by the fact that the color $c$ appears 'at rest' in the reference frame $I$.

Carrying on the analogy with mechanics, we will call $o_{1}=\left(c_{1}, I_{1}\right)$ and $o_{2}=\left(c_{2}, I_{2}\right)$ two inertial observers. Given $i, j=1,2, i \neq j, c_{j}$ will be described by $o_{i}$ with a color $c_{j}^{i}=\left(\alpha, \mathbf{v}_{i j}\right)$, for a certain vector $\mathbf{v}_{i j}$ such that $\left\|\mathbf{v}_{i j}\right\| \leq \Sigma$ and, by definition of observer, $c_{i}^{i}=(\alpha, \mathbf{0})$, being $c_{i}^{i}$ the coordinate representation of $c_{i}$ w.r.t. $I_{i}, i=1,2$. Given two inertial observers $o_{1}$ and $o_{2}$, we denote by $\left(\alpha^{1}, x^{1}, y^{1}\right)$ the coordinates of the reference frame $I_{1}$ and by $\left(\alpha^{2}, x^{2}, y^{2}\right)$ the coordinates of the reference frame $I_{2}$.

\subsection{Yilmaz experiments}

Thanks to the nomenclature just introduced, we are now able to give a concise description of Yilmaz experiments, for the original description see [26, 18] or the appendix.

Yilmaz considers only the case of one non-zero component of the $\mathbf{v}$ vector, i.e. $\mathbf{v}_{c_{1}}=\left(v_{c_{1}}, 0\right)$, $\mathbf{v}_{c_{2}}=\left(v_{c_{2}}, 0\right)$, and $\mathbf{v}_{12}=\left(v_{12}, 0\right)$.

The result of the first experiment can be expressed by the following equalities:

$$
\mathbf{v}_{c_{2}^{1}}=\mathbf{v}_{12}, \quad \mathbf{v}_{c_{1}^{2}}=-\mathbf{v}_{12} .
$$

Since, for $i, j=1,2, i \neq j$, the saturation of $c_{i}$ is null in the reference frame $I_{i}$ and different from zero in the reference frame $j$, eq. (19) clearly shows that color perception is a relativistic phenomenon.

Also the second experiment involves two inertial observers $o_{1}=\left(c_{1}, I_{1}\right)$ and $o_{2}=\left(c_{2}, I_{2}\right)$, both describing the same color $c$. The result of the second experiment can be summarized as follows:

$$
\mathbf{v}_{c^{1}}=(\Sigma, 0) \Longrightarrow \mathbf{v}_{c^{2}}=(\Sigma, 0)
$$

i.e., colors with limiting saturation are perceived as such by all inertial observers.

As already mentioned, the third experiment is meant to mimic the relativistic aberration effect. Once again, it involves two inertial observers $o_{1}$ and $o_{2}$, both observing the same color $c$, different from the previous one. The result of the third experiment is the following:

$$
\mathbf{v}_{c^{1}}=(0, \Sigma) \Longrightarrow \mathbf{v}_{c^{2}}=(-\Sigma \sin \varphi, \Sigma \cos \varphi)
$$

with $\sin \varphi=v_{12} / \Sigma$. As we are going to see, this experiment is crucial for the derivation of the colorimetric Lorentz transformations.

\subsection{Yilmaz derivation of colorimetric Lorentz transformations}

We explain now how to obtain the colorimetric Lorentz transformations from eqs. $(19,2)$ and 21]. In [26] the coordinate change between $o_{1}$ and $o_{2}$ is supposed to be linear. As shown in detail is [18, when we take into account the specific choices made by Yilmaz, the coordinate change is given by:

$$
\left(\begin{array}{l}
\alpha^{2} \\
x^{2} \\
y^{2}
\end{array}\right)=\left(\begin{array}{ccc}
a_{11} & a_{12} & 0 \\
a_{21} & a_{22} & 0 \\
0 & 0 & 1
\end{array}\right)\left(\begin{array}{l}
\alpha^{1} \\
x^{1} \\
y^{1}
\end{array}\right) .
$$

Consequently, we have:

$$
\frac{x_{2}}{\alpha_{2}}=\frac{a_{21} \alpha_{1}+a_{22} x_{1}}{a_{11} \alpha_{1}+a_{12} x_{1}}, \quad \frac{x_{1}}{\alpha_{1}}=\frac{-a_{21} \alpha_{2}+a_{11} x_{2}}{a_{22} \alpha_{2}-a_{12} x_{2}} .
$$

The two equalities of eq. 19 are equivalent to:

$$
\frac{a_{21}}{a_{11}}=-v_{12}, \quad \frac{-a_{21}}{a_{22}}=v_{12} .
$$


This shows that: $a_{11}=a_{22}$ and $a_{21}=-v_{12} a_{22}$.

The result of the second experiment, eq. 200, is equivalent to:

$$
\Sigma=\frac{a_{21}+a_{22} \Sigma}{a_{11}+a_{12} \Sigma},
$$

which gives: $a_{12}=-\left(v_{12} a_{22}\right) / \Sigma^{2}$.

From the third experiment, eq. (21), we have:

$$
-\tan \varphi=\frac{a_{21} \alpha_{1}+a_{22} x_{1}}{y_{1}}=\frac{a_{21}}{\Sigma} .
$$

Since $\sin \varphi=v_{12} / \Sigma$, this implies:

$$
a_{22}=\frac{1}{\sqrt{1-\left(v_{12} / \Sigma\right)^{2}}} .
$$

Finally:

$$
\left(\begin{array}{l}
\alpha^{2} \\
x^{2} \\
y^{2}
\end{array}\right)=\left(\begin{array}{ccc}
\frac{1}{\sqrt{1-\left(v_{12} / \Sigma\right)^{2}}} & \frac{-v_{12} / \Sigma^{2}}{\sqrt{1-\left(v_{12} / \Sigma\right)^{2}}} & 0 \\
\frac{-v_{12}}{\sqrt{1-\left(v_{12} / \Sigma\right)^{2}}} & \frac{1}{\sqrt{1-\left(v_{12} / \Sigma\right)^{2}}} & 0 \\
0 & 0 & 1
\end{array}\right)\left(\begin{array}{l}
\alpha^{1} \\
x^{1} \\
y^{1}
\end{array}\right),
$$

which can be recognized to be a Lorentz boost along the $x$-direction. It is worth noticing that the derivation of these colorimetric Lorentz transformations proposed by Yilmaz relies only on information given by the $\mathbf{v}$-component of colors, the only one appearing in eqs. $(19), 20)$ and 21). In the quantum framework, these $\mathbf{v}$-components correspond to perceptual chromatic vectors that will be introduced in 5.1

\subsection{Issues about Yilmaz approach}

Without calling into question the great originality of Yilmaz's ideas and the relevance of his results, we deem necessary to underline some issues about the approach that we have reported above. As mentioned before, the derivation of the colorimetric Lorentz transformations is essentially based on the following assumptions:

- the space of perceived colors is the cone, and, in particular, there exists a limiting saturation $\Sigma$;

- the coordinate changes between inertial observers are linear transformations;

- the results obtained from the three experiments are considered as valid.

However, no experimental result, nor apparatus description is available in [26] and this naturally raises doubts about the actual implementation of the three experiments. Furthermore, while the results of the first two experiments is plausible, the outcome of the third seems completely illusory. In fact, Yilmaz defines the limiting saturation of a color $c=(\alpha, x, y) \in \widetilde{\mathcal{C}}$ as a value $\Sigma$ of $\|v\|$ that cannot be perceptually matched with that of any Munsell chip, thus, while this definition permits to identify the limiting saturation of a color, it does not allow its measurement. As a consequence, eq. (21), with its precise analytical form, seems to be an ad-hoc formula used to single out the colorimetric Lorentz transformations (28), more than the real outcome of a psycho-physical experiment.

It may be tempting to adopt a more conventional approach to obtain the desired transformations starting, for instance, from the fact that there exists a limiting saturation invariant under observer changes and that the color space is isotropic and homogeneous. However, to go further, it is necessary to introduce an analogue of the Minkowski metric, which Yilmaz precisely circumvents. One may choose to follow the standard path used in special relativity, see e.g. [14, 15, to justify the existence of such a metric. However, while the assumptions that go along with this approach rely on a solid experimental basis for what concerns the Minkowski spacetime, they are far from being either obvious or simple to be tested for the space of perceived colors. For this 
reason, we consider a better solution to follow less conventional, but fully equivalent, approaches to special relativity as, e.g., that of the remarkable Mermin's paper [16, whose main focus is the Einstein-Poincaré velocity addition law and not Lorentz transformations. As mentioned in the introduction, this alternative approach seems more suitable because the colorimetric effects reported by Yilmaz involve the sole $\mathbf{v}$-components or equivalently the sole perceptual chromatic vectors.

\section{Einstein-Poincaré's addition law for chromatic vectors and the formalization of Yilmaz first two experimental results}

In this section we show that the outcomes of the first two experiments quoted by Yilmaz in his model can be rigorously derived from the fact that the so-called perceptual chromatic vectors, that will be introduced in subsection 5.1, satisfy the Einstein-Poincaré addition law.

In order to show in the clearest way how to obtain the results stated above, we start by introducing the concept of perceptual chromatic vector, alongside with several other definitions of chromatic attributes.

\subsection{The nomenclature of quantum perceptual color attributes}

We recall that a perceptual color $c$ is an element of the trichromacy cone $\mathcal{C}$, i.e. explicitly $c=(\alpha, \mathbf{v})$ with $\alpha^{2}-\|\mathbf{v}\|^{2} \geq 0$ and $\alpha \geq 0$. The positive real $\alpha$ is called the magnitud $\xi^{2}$ of $c$. Since the cone $\mathcal{C}$ is self-dual, then $c$ can also be considered as an element of the dual cone $\mathcal{C}^{*}$.

The case when $c$ has magnitude $\alpha=1 / 2$ is special, in fact, as previously seen, thanks to the isomorphism defined in eq. (3), $c$ can naturally be associated to a density matrix representing its state. For this reason, in the sequel, a color with magnitude $1 / 2$ will be called a perceptual color state. To emphasize that a perceptual color $c$ is a perceptual color state we add the subscript $s$, so that the symbol $c_{s}$ will denote a color $(1 / 2, \mathbf{v})$, with $\|\mathbf{v}\| \leq 1 / 2$.

We can associate to any other color $c$ with arbitrary magnitude $\alpha \geq 0$ a density matrix by considering the projection obtained by dividing $c$ with respect to twice its first component, i.e. $c / 2 \alpha=(1 / 2, \mathbf{v} / 2 \alpha)$, which belongs to $\mathcal{D}_{1 / 2}=\{c \in \mathcal{C}, \alpha=1 / 2\}$, the set of all perceptual colors whose magnitude is fixed to $1 / 2$.

The vector $\mathbf{v}_{c} \in \mathcal{D}_{1 / 2}$ is called the perceptual chromatic vector of the color $c$ because it carries only information about the chromatic attributes of $c$ and not about its magnitude.

By using eq. (5), we can associate a density matrix to every perceptual chromatic vector $\mathbf{v}_{c}=\left(v_{c, 1}, v_{c, 2}\right) \in \mathcal{D}_{1 / 2}$ simply by considering $2 \mathbf{v}_{c} \in \mathcal{D}$, which allows us to write:

$$
\rho\left(2 \mathbf{v}_{c}\right)=\frac{1}{2}\left(\begin{array}{cc}
1+2 v_{c, 1} & 2 v_{c, 2} \\
2 v_{c, 2} & 1-2 v_{c, 1}
\end{array}\right) .
$$

For every perceptual color $c \in \mathcal{C}$, the density matrix $\rho\left(2 \mathbf{v}_{c}\right)$ characterizes the perceptual chromatic state of $c$. The difference between a color and a chromatic state is represented by the fact that, in the first case, the density matrix associated to a color $c$ with magnitude $1 / 2$ contains all the information about the state of $c$, magnitude included, which is not the case for a chromatic state, where the magnitude $\alpha$ of $c$ does not play any role.

Two noticeable conditions about perceptual chromatic states can be singled out: the first is when the density matrix $\rho\left(2 \mathbf{v}_{c}\right)$ describes a pure perceptual chromatic state, in this case, $c \in \mathcal{C}$ is said to be a pure perceptual color. This condition is equivalent to $\left\|\mathbf{v}_{c}\right\|=1 / 2$, so that pure perceptual colors are in one-to-one correspondence with the points of the perimeter of the disk $\mathcal{D}_{1 / 2}$. The second noticeable condition is when $\rho\left(2 \mathbf{v}_{c}\right)$ describes the state of maximal von Neumann

\footnotetext{
${ }^{2}$ We prefer not to use the term lightness because of possible confusion. See for instance [13] for a discussion on the meaning of this word.
} 
entropy, in this case $c \in \mathcal{C}$ is said to be an achromatic perceptual color. Since the condition $\rho\left(2 \mathbf{v}_{c}\right)=\rho_{0}=I d_{2} / 2$ is equivalent to $v_{c, 1}=v_{c, 2}=0$, it follows that the center of the disk $\mathcal{D}_{1 / 2}$ represents achromatic perceptual colors.

We now introduce the chromaticity descriptors, that we will call purities and quantities. For the sake of simplicity, we will consider only colors $c$ whose perceptual chromatic vectors are of the form $\mathbf{v}_{c}=\left(v_{c}, 0\right)$ with $-1 / 2 \leq v_{c} \leq 1 / 2$. The two chromatic vectors $\mathbf{v}_{+}=(1 / 2,0)$ and $\mathbf{v}_{-}=(-1 / 2,0)$ are pure opponent chromatic vectors. Given a color $c$, its chromatic vector $\mathbf{v}_{c}$ divides the segment connecting $\mathbf{v}_{-}$and $\mathbf{v}_{+}$(extremes excluded) in two parts, whose lengths are denoted by $p^{-}(c)$ and $p^{+}(c)$, where:

$$
p^{-}(c)=\frac{1}{2}-v_{c}=\frac{1-2 v_{c}}{2},
$$

will be called the - purity of $c$ and

$$
p^{+}(c)=v_{c}-\left(-\frac{1}{2}\right)=\frac{1+2 v_{c}}{2},
$$

will be called the + purity of $c$. The sum of the - and + purity of $c$ is 1 , so $\mathbf{v}_{c}$ can be written as the convex combination of the pure opponent chromatic vectors $\mathbf{v}_{-}$and $\mathbf{v}_{+}$with weights given by $p^{-}$ and $p^{+}$, respectively, i.e. $\mathbf{v}_{c}=p^{-}(c) \mathbf{v}_{-}+p^{+}(c) \mathbf{v}_{+}$. The term 'purity' is particularly appropriate, not only because it involves the pure opponent chromatic vectors, but also because it is reminiscent of the same term appearing in classical CIE (Commission International de l'Éclairage) colorimetry. Indeed, also the definition of 'excitation purity' $p_{e}$ of a color $c$ carries the information about its position on a straight line, precisely the one joining the equienergy point $w$ (achromatic color) of the CIE 1931 chromaticity diagram with the so-called dominant wavelength of $c$ (represented by a point belonging to the border of the chromaticity diagram). See [25] for more details.

Let also denote

$$
r(c)=\frac{p^{-}(c)}{p^{+}(c)}=\frac{1-2 v_{c}}{1+2 v_{c}},
$$

the purity ratio of the color $c$. We have:

$$
v_{c}=\frac{1}{2}\left(\frac{p^{+}(c)-p^{-}(c)}{p^{+}(c)+p^{-}(c)}\right) .
$$

It is obvious that, given two colors $c$ and $d$, we have:

$$
\mathbf{v}_{c}=\mathbf{v}_{d} \Longleftrightarrow p^{+}(c)=p^{+}(d) \Longleftrightarrow p^{-}(c)=p^{-}(d) .
$$

Two colors with the same purity differ only by their magnitude. For this reason, it is useful to define a color attribute analogue to purity but which takes into account also the magnitude information that has been lost after the projection on $\mathcal{D}_{1 / 2}$. This is done as follows: we define the

- quantity of a color $c$ by:

$$
q^{-}(c)=2 \alpha_{c} p^{-}(c)=\alpha_{c}\left(1-2 v_{c}\right)
$$

and similarly the + quantity by:

$$
q^{+}(c)=2 \alpha_{c} p^{+}(c)=\alpha_{c}\left(1+2 v_{c}\right),
$$

where $\alpha_{c}$ is the magnitude of $c$. Of course, perceptual colors with magnitude equal to $1 / 2$, i.e. percpetual color states, are characterized by the fact that their purities and quantities coincide.

\subsection{Einstein-Poincaré addition law for perceptual chromatic vectors and Yilmaz first two experiments}

Now we discuss our main issue: how can we describe a given color $c$ relatively to another given color $d$ ? One intuitive way to do it is to compare $q^{-}(c)$ with $q^{-}(d)$ and $q^{+}(c)$ with $q^{+}(d)$, that is 
to compare their - and + quantities. For this, we can consider the quantity ratios:

$$
s^{+}(c, d)=\frac{q^{+}(c)}{q^{+}(d)} \quad \text { and } \quad s^{-}(c, d)=\frac{q^{-}(c)}{q^{-}(d)} .
$$

If we only know the numerical values of $q^{ \pm}(c), q^{ \pm}(d)$ and not their explicit expressions (35) and (36), then using the quantity ratio to compare $c$ and $d$ makes sense only if $d$ is a perceptual color state. In fact, and to take an example, if $c$ and $d$ are two perceptual colors with the same chromatic vector, the ratio, for instance, $s^{+}(c, d)$ does not give any information about the description of $c$ relatively to $d$ since we do not know the magnitude of $d$.

Let us consider two arbitrary colors $c$ and $d$ whose magnitudes and chromatic vectors are respectively $\alpha_{c}$ and $\alpha_{d}$, and $\mathbf{v}_{c}$ and $\mathbf{v}_{d}$, with $v_{c}>v_{d}$. We have:

$$
s^{+}(c, d)=\frac{\alpha_{c} p^{+}(c)}{\alpha_{d} p^{+}(d)} \quad \text { and } \quad s^{-}(c, d)=\frac{\alpha_{c} p^{-}(c)}{\alpha_{d} p^{-}(d)} .
$$

In order to describe $c$ with respect to $d$, we have to perform quantity ratios between $c$ and $d_{s}$, this latter being the color state whose chromatic vector equals $\mathbf{v}_{d}$. We write:

$$
s^{+}(c, d)=\frac{q^{+}(c)}{2 \alpha_{d} p^{+}\left(d_{s}\right)}=\frac{q^{+}(c)}{2 \alpha_{d} q^{+}\left(d_{s}\right)}=\frac{1}{2 \alpha_{d}} s^{+}\left(c, d_{s}\right),
$$

and the same with the minus sign.

Now we arrive to the key definition of a chromatic vector, that we will denote with $\mathbf{v}_{d}^{c}$, that describes the color $c$ with respect to $d$. In order to do that, we take inspiration from eq. (33) with quantity ratios playing the role of purities, thus obtaining:

$$
v_{d}^{c}=\frac{1}{2}\left(\frac{s^{+}\left(c, d_{s}\right)-s^{-}\left(c, d_{s}\right)}{s^{+}\left(c, d_{s}\right)+s^{-}\left(c, d_{s}\right)}\right) .
$$

We have:

$$
v_{d}^{c}=\frac{1}{2}\left(\frac{q^{+}(c) q^{-}(d)-q^{-}(c) q^{+}(d)}{q^{+}(c) q^{-}(d)+q^{-}(c) q^{+}(d)}\right)
$$

that is:

$$
v_{d}^{c}=\frac{1}{2}\left(\frac{p^{+}(c) p^{-}(d)-p^{-}(c) p^{+}(d)}{p^{+}(c) p^{-}(d)+p^{-}(c) p^{+}(d)}\right) .
$$

Now we compute:

$$
\frac{v_{c}-v_{d}}{1-4 v_{c} v_{d}}=\frac{\frac{1}{2}\left(\frac{p^{+}(c)-p^{-}(c)}{p^{+}(c)+p^{-}(c)}\right)-\frac{1}{2}\left(\frac{p^{+}(d)-p^{-}(d)}{p^{+}(d)+p^{-}(d)}\right)}{1-\frac{p^{+}(c)-p^{-}(c)}{p^{+}(c)+p^{-}(c)} \cdot \frac{p^{+}(d)-p^{-}(d)}{p^{+}(d)+p^{-}(d)}} .
$$

We have:

$$
\frac{v_{c}-v_{d}}{1-4 v_{c} v_{d}}=\frac{1}{2}\left(\frac{\left(p^{+}(d)+p^{-}(d)\right)\left(p^{+}(c)-p^{-}(c)\right)-\left(p^{+}(d)-p^{-}(d)\right)\left(p^{+}(c)+p^{-}(c)\right)}{\left(p^{+}(c)+p^{-}(c)\right)\left(p^{+}(d)+p^{-}(d)\right)-\left(p^{+}(c)-p^{-}(c)\right)\left(p^{+}(d)-p^{-}(d)\right)}\right)
$$

This shows that:

$$
\mathbf{v}_{d}^{c}=\frac{\mathbf{v}_{c}-\mathbf{v}_{d}}{\mathbf{1}-4 \mathbf{v}_{c} \mathbf{v}_{d}}
$$

or equivalently:

$$
\mathbf{v}_{c}=\frac{\mathbf{v}_{d}^{c}+\mathbf{v}_{d}}{1+4 \mathbf{v}_{d}^{c} \mathbf{v}_{d}} .
$$

Equations 45 and 46 are the exact analogue of the Einstein-Poincaré addition law of special relativity written with our nomenclature with the only exception of the factor 4 in eq. (46) which is due to the fact that chromatic vectors have norms less or equal to $1 / 2$, i.e. that in our model the limiting saturation $\Sigma$ is equal to $1 / 2$. 
Thanks to eqs. 45) and (46), the proof of the fact that the first two outcomes of Yilmaz's experiments can be derived in a purely theoretical manner is extremely simple. In fact, eq. 45 implies that $\mathbf{v}_{c}^{d}=-\mathbf{v}_{d}^{c}$, which is nothing but an alternative way of writing eq. $\sqrt{19}$, i.e. the result of the first Yilmaz experiment. Moreover, if we introduce $\mathbf{v}_{d}^{c}=(\Sigma, 0)$ in eq. 46, , then we obtain $\mathbf{v}_{c}=(\Sigma, 0)$, which is the precisely eq. 20 written with the symbols of the present section, i.e. the result of Yilmaz's second experiment.

\section{A theoretically and experimentally coherent distance on the space of perceptual chromatic vectors: the Hilbert metric}

In this section we prove that, quite remarkably, the Einstein-Poincaré additivity law satisfied by perceptual chromatic vectors permits to coherently equip the space of such vectors with the socalled Hilbert metric. In subsection 6.1] we show that this metric is compatible with the results of well-established psycho-visual experiments.

Let us start by recalling that, given four collinear points $a, p, q$, and $b$ of $\mathbb{R}^{2}$, with $a \neq p$ and $q \neq b$, the cross ratio $[a, p, q, b]$ is defined by $[5]$ :

$$
[a, p, q, b]=\frac{\|q-a\|}{\|p-a\|} \cdot \frac{\|p-b\|}{\|q-b\|},
$$

where $\|\cdot\|$ denotes the Euclidean norm. Given two points $p$ and $q$ of the closed disk $\mathcal{D}_{1 / 2}$ such that the points $(-1 / 2,0)=a_{-}, p, q$, and $(1 / 2,0)=a_{+}$are collinear with the segment $[p, q]$ contained in the segment $\left[a_{-}, a_{+}\right]$, the $\mathcal{D}_{1 / 2}$-Hilbert distance $d_{H}(p, q)$ is given by [5]:

$$
d_{H}(p, q)=\frac{1}{2} \ln \left[a_{-}, p, q, a_{+}\right],
$$

where the choice of the points involved in the cross ratio above guarantees that the argument of ln is strictly positive.

We consider now three chromatic vectors $\mathbf{v}_{c}, \mathbf{v}_{d}$ and $\mathbf{v}_{d}^{c}$ of $\mathcal{D}_{1 / 2}$ with $\mathbf{v}_{c}=\left(v_{c}, 0\right), \mathbf{v}_{d}=\left(v_{d}, 0\right)$ and $\mathbf{v}_{d}^{c}=\left(v_{d}^{c}, 0\right)$. We have the following elementary result (see for instance [9] for related topics).

Proposition 6.1 With the notations introduced above, it holds that:

$$
d_{H}\left((0,0),\left(v_{d}^{c}, 0\right)\right)=d_{H}\left(\left(v_{d}, 0\right),\left(v_{c}, 0\right)\right) \Longleftrightarrow v_{c}=\frac{v_{d}^{c}+v_{d}}{1+4 v_{d}^{c} v_{d}} .
$$

Proof. By definition, the equality $d_{H}\left((0,0),\left(v_{d}^{c}, 0\right)\right)=d_{H}\left(\left(v_{c}, 0\right),\left(v_{d}, 0\right)\right)$ holds if and only if $\left[a_{-},(0,0),\left(v_{d}^{c}, 0\right), a_{+}\right]=\left[a_{-},\left(v_{d}, 0\right),\left(v_{c}, 0\right), a_{+}\right]$. Equivalently:

$$
d_{H}\left((0,0),\left(v_{d}^{c}, 0\right)\right)=d_{H}\left(\left(v_{c}, 0\right),\left(v_{d}, 0\right)\right) \Longleftrightarrow \frac{1 / 2-v_{c}}{1 / 2+v_{c}}=\frac{1 / 2-v_{d}^{c}}{1 / 2+v_{d}^{c}} \cdot \frac{1 / 2-v_{d}}{1 / 2+v_{d}} .
$$

By direct computation, it can be checked that this last equation is equivalent to eq. 49.

The colorimetric interpretation of the relation:

$$
d_{H}\left(\mathbf{0}, \mathbf{v}_{d}^{c}\right)=d_{H}\left(\mathbf{v}_{d}, \mathbf{v}_{c}\right) \Longleftrightarrow \mathbf{v}_{c}=\frac{\mathbf{v}_{d}^{c}+\mathbf{v}_{d}}{1+4 \mathbf{v}_{d}^{c} \mathbf{v}_{d}}
$$

is the following. The vector $\mathbf{v}_{d}^{c}$ appears in the relativistic sum equation expressed by (46) together

with the chromatic vectors $\mathbf{v}_{c}$ and $\mathbf{v}_{d}$ if and only if the Hilbert length $d_{H}\left(\mathbf{0}, \mathbf{v}_{d}^{c}\right)$ of $\mathbf{v}_{d}^{c}$ is equal to the Hilbert distance between $\mathbf{v}_{c}$ and $\mathbf{v}_{d}$. Since $\mathbf{v}_{d}^{c}$ describes the color $c$ with respect to the 
color $d$, this result implies that the Hilbert distance is a mathematically coherent candidate for a perceptual metric of chromatic attributes.

A geometric representation of this result is provided by the so-called Chasles theorem on cross ratios of cocyclic points, see Figure 2, which provides a graphical method to construct the relativistic sum of two vectors in one dimension. Although elementary, this result reveals a meaningful link between the Einstein-Poincaré addition law of chromatic vectors and the Hilbert metric, which, on $\mathcal{D}_{1 / 2}$, coincides precisely with the Klein hyperbolic metric defined by:

$$
d s_{\mathcal{D}_{1 / 2}}^{2}=\frac{\left(1 / 4-v_{2}^{2}\right) d v_{1}^{2}+2 v_{1} v_{2} d v_{1} d v_{2}+\left(1 / 4-v_{1}^{2}\right) d v_{2}^{2}}{\left(1 / 4-\|v\|^{2}\right)^{2}} .
$$

The geodesics with respect to this metric are straight chords of $\mathcal{D}_{1 / 2}$.

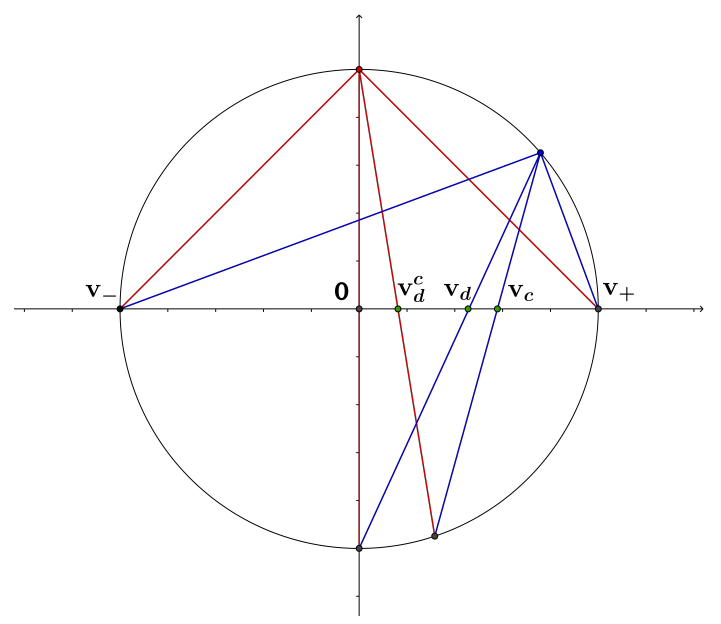

Figure 2: Illustration of the result of Prop. 6.1 by Chasles theorem on the cross ratios of cocyclic points. $\mathbf{v}_{c}, \mathbf{v}_{d}$ and $\mathbf{v}_{d}^{c}$ satisfy eq. (51).

\subsection{Compatibility of the Hilbert metric with psycho-visual experimen- tal data}

Now we address the important issue of the compatibility between the Hilbert metric on $\mathcal{D}_{1 / 2}$ and psychovisual measurements. This is not an easy task because of two reasons: firstly, experimental data on color perception are very scarce, secondly, psychovisual measurements are always affected by subjective variations which imply the use of averaging procedures that inevitably reduce the measure accuracy. The only psychovisual results consistent with our framework that we were able to find are those reported in 4 and [6. The authors conducted their tests with the help of the standard CIE illuminants $C$ (near-daylight, $\left(x_{C}, y_{C}\right)=(0.3125,0.3343)$ ) and $A$ (tungsten, $\left.\left(x_{A}, y_{A}\right)=(0.4475,0.4084)\right)$ and added a third one, denoted with $G$ (greenish, $\left.\left(x_{G}, y_{G}\right)=(0.3446,0.4672)\right)$. The values $(x, y)$ represent the CIE $x y Y$ chromaticity coordinates of $C, A$ and $G$, respectively, Fig. 6.1 shows their position in the chromaticity diagram. In what follows, observers adapted to the illuminants $C, A$ and $G$, respectively, will be denoted by $o_{1}=(c, C)$, $o_{2}=(a, A)$ and $o_{3}=(g, G)$. A haploscope is used to compare the color perception of one eye always adapted to the illuminant $C$ and the other eye adapted to $C, A$ and $G$.

Fig. 6.1 shows, in the $x y Y$ diagram, three families of curves obtained by the tests performed in [6]:

- the first is composed by three contours surrounding $C$ that correspond to color stimuli with fixed Munsell value, different hue but with the same perceived Munsell chroma in $\{2,4,8\}$. By normalizing these data between 0 and 0.5 we obtain $\{0.1,0.2,0.4\}$, which are the norms of the chromatic vectors $\mathbf{v}_{c}^{1}$ of the colors associated to the corresponding stimuli observed by $o_{1}$; 
- the second and the third are given by two contours surrounding $A$, resp. $G$, that correspond to colors $c$ with varying hues and whose Munsell chroma belong to the set $\{2,4\}$. The chromatic vectors $\mathbf{v}_{c}^{2}$, resp. $\mathbf{v}_{c}^{3}$, of these colors observed from $o_{2}$, resp. $o_{3}$, have norms belonging to the set $\{0.1,0.2\}$.

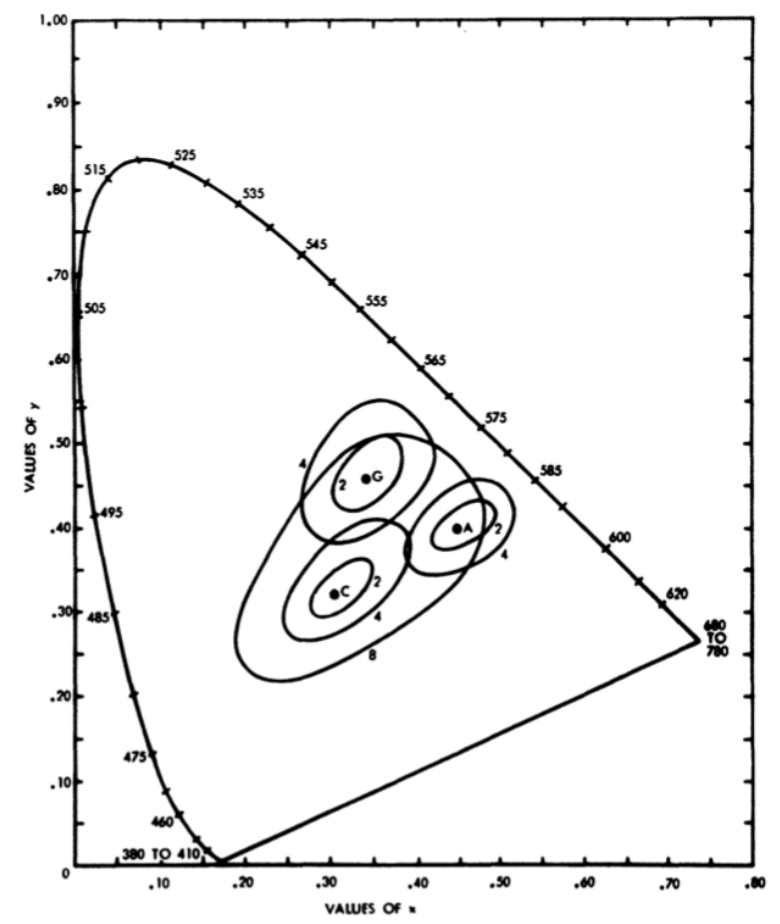

Figure 3: The iso Munsell chroma contours found by [6] in the $x y Y$ diagram.

As discussed above, the psychovisual data reported in [4] and [6] are only averaged, thus, the only kind of information that we have from Fig. 6.1 is, for example, that the $x y Y$ coordinates of standard illuminant $A$ are between the curves of chroma 4 and 8 of the observer $o_{1}$. Thus, the norm of the chromatic vectors is not possible to achieve with accuracy. However, in order to test our mathematical theory, as a first approximation, we perform a linear interpolation from the data appearing in the figure, which gives $\left\|\mathbf{v}_{a}^{1}\right\| \simeq 6.76 / 20=0.338$.

In Fig. 4(a), we denote by $F$ and $F^{\prime}$ the $x y Y$ coordinates of the points in the $x y Y$ diagram obtained by the intersection between the line connecting $A$ and $C$ with the iso-chroma contours for $o_{1}$ and $o_{2}$, respectively. The color $F$ is perceived by $o_{1}$ as having a chromatic vector $\mathbf{v}_{f}^{1}$ with norm $\left\|\mathbf{v}_{f}^{1}\right\|=0.2$. By construction, we determine $F^{\prime}$, the color perceived by $o_{2}$ with chromatic vector $\mathbf{v}_{f^{\prime}}^{2}$ such that $\mathbf{v}_{f^{\prime}}^{2}=\mathbf{v}_{f}^{1}$. Again, by linear interpolation, the norm of the chromatic vector $\mathbf{v}_{f^{\prime}}^{1}$ corresponding to the color $F^{\prime}$ perceived by $o_{1}$, is approximated by $\left\|\mathbf{v}_{f^{\prime}}^{1}\right\| \simeq 3.76 / 20=0.188$. Fig. 4(b) shows all the chromatic vectors in the disk $\mathcal{D}_{1 / 2}$.

One can easily check, as illustrated by Chasles theorem, that:

$$
d_{H}\left(\mathbf{v}_{f}^{1}, \mathbf{v}_{c}^{1}\right)=d_{H}\left(\mathbf{v}_{f^{\prime}}^{2}, \mathbf{v}_{a}^{2}\right)=d_{H}\left(\mathbf{v}_{f^{\prime}}^{1}, \mathbf{v}_{a}^{1}\right) .
$$

The same reasoning applied to the situation depicted in Fig. 5(a), where the points $F 2$ and $F 2^{\prime}$ belong to another iso-chroma contour, leads to:

$$
d_{H}\left(\mathbf{v}_{f 2}^{1}, \mathbf{v}_{c}^{1}\right)=d_{H}\left(\mathbf{v}_{f 2^{\prime}}^{2}, \mathbf{v}_{a}^{2}\right)=d_{H}\left(\mathbf{v}_{f 2^{\prime}}^{1}, \mathbf{v}_{a}^{1}\right),
$$

see Fig. 5(b). 


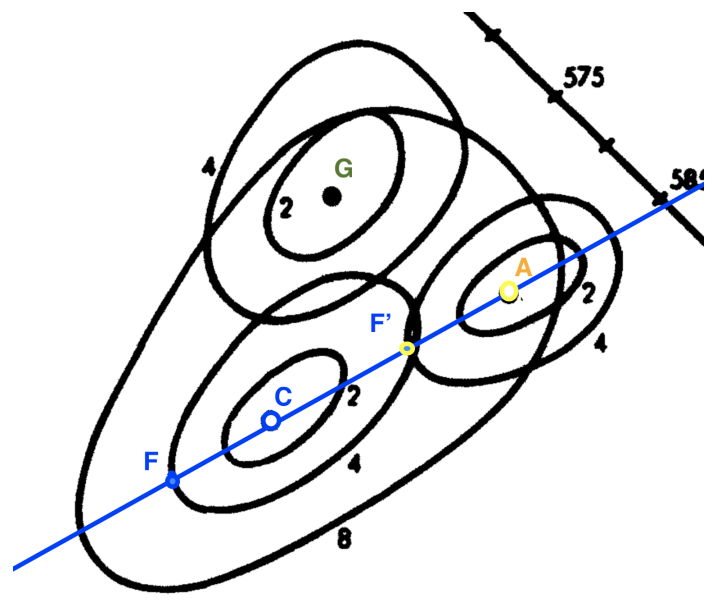

(a) The illuminants $C$ and $A$ and the colors $F$ and $F^{\prime}$ in the $x y Y$ diagram.

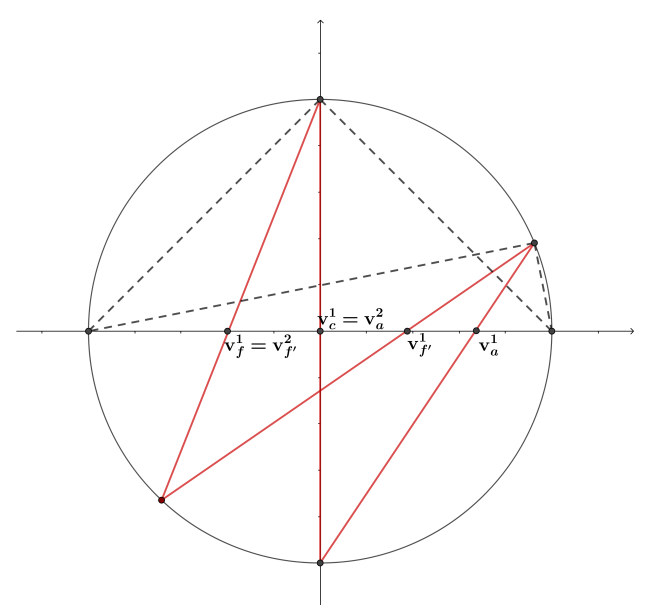

(b) Illustration of the equalities of eq. 53 in the disk $\mathcal{D}_{1 / 2}$.

Figure 4: Invariance of the Hilbert distance under observer changes: illuminants $C$ and $A$, and colors $F$ and $F^{\prime}$.

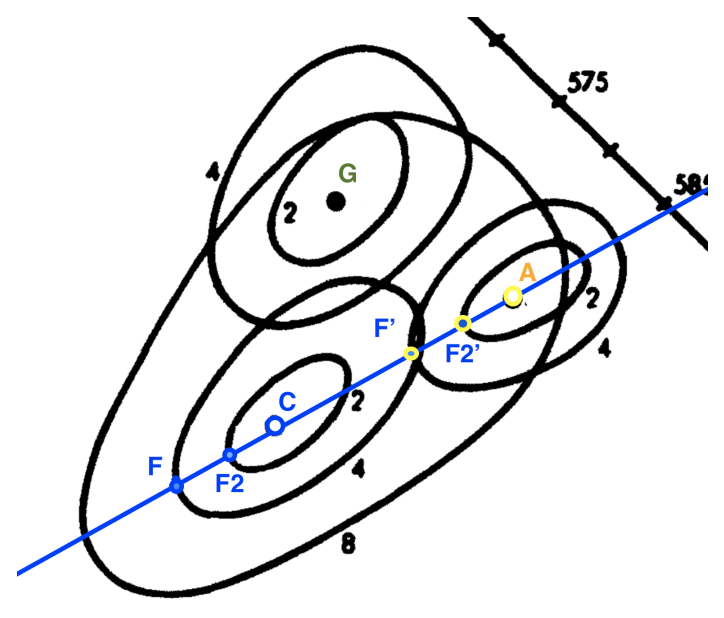

(a) The illuminants $C$ and $A$ and the colors $F$ and $F^{\prime}$, and $F 2$ and $F 2^{\prime}$ in the CIE xyY diagram.

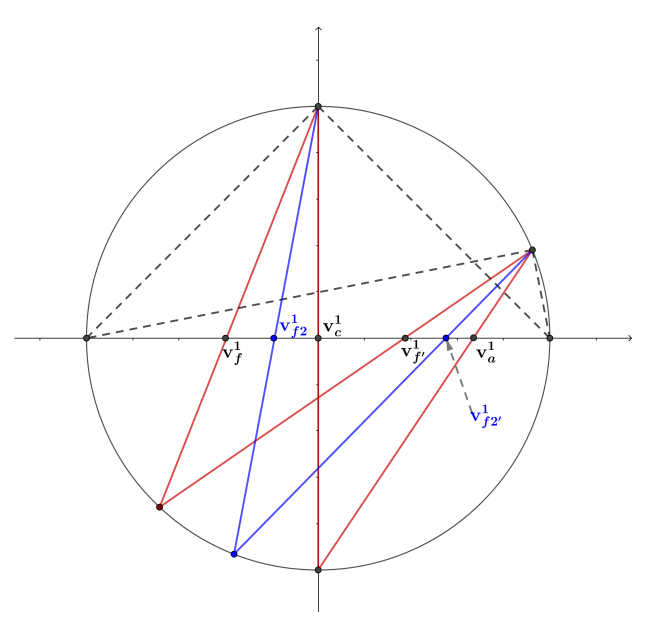

(b) Illustration of the equalities of Eq. 54 in the disk $\mathcal{D}_{1 / 2}$.

Figure 5: Invariance of the Hilbert distance under observer changes: illuminants $C$ and $A$, and colors $F, F^{\prime}, F 2$, and $F 2^{\prime}$.

Finally, we consider the quite more complicated situation depicted in Fig. 6(a). It is precised in 4 that 'A change from a blue $(C)$ adaptation to a yellow $(A)$ adaptation shows vectors running in a blue-yellow direction, a change from a blue $(C)$ adaptation to a green $(G)$ adaptation shows vectors running in a blue-green direction.' This means that the angle between $\mathbf{v}_{a}^{1}$ and $\mathbf{v}_{g}^{1}$ is equal to $\pi / 4$. From Fig. 6(a) we can approximate the norm of the chromatic vector $\mathbf{v}_{q}^{1}:\left\|\mathbf{v}_{g}^{1}\right\| \simeq 0.32$. The chromatic vectors $\mathbf{v}_{h}^{1}$ and $\mathbf{v}_{h^{\prime \prime}}^{3}$ of the two colors $H$ and $H^{\prime \prime}$ marked on Fig. 6(a) are equal. Once again, one can easily check that:

$$
d_{H}\left(\mathbf{v}_{h}^{1}, \mathbf{v}_{c}^{1}\right)=d_{H}\left(\mathbf{v}_{h^{\prime \prime}}^{3}, \mathbf{v}_{g}^{3}\right)=d_{H}\left(\mathbf{v}_{h^{\prime \prime}}^{1}, \mathbf{v}_{g}^{1}\right),
$$

see Fig. 6(b) 


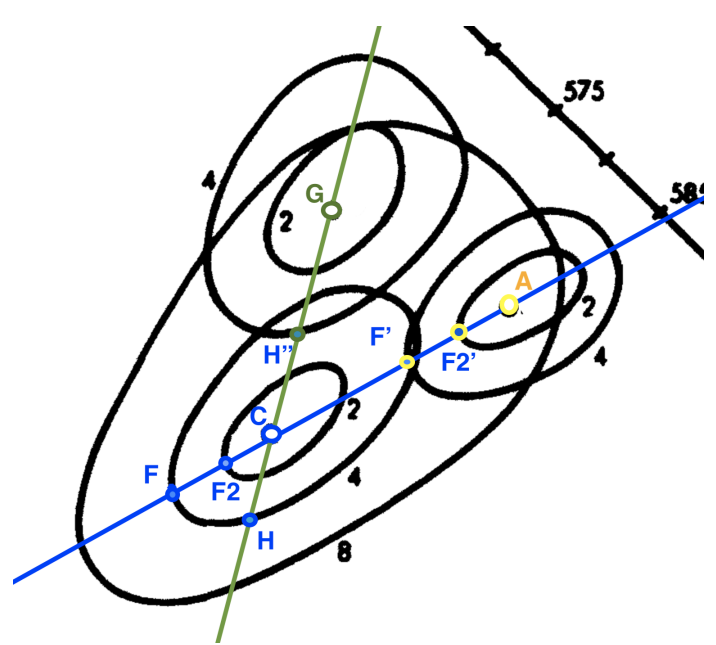

(a) The three illuminants $C, A$ and $G$, and the colors $F$ and $F^{\prime}, F 2$ and $F 2^{\prime}$, and $H$ and $H^{\prime \prime}$ in the $x y Y$ diagram.

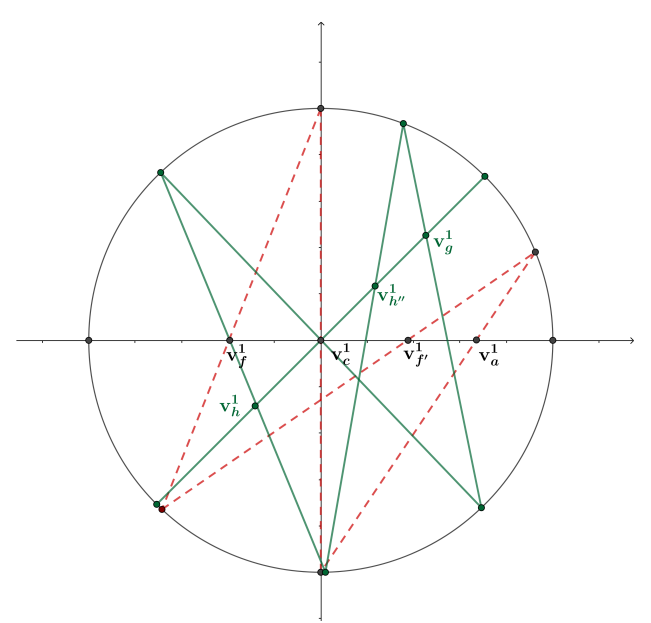

(b) Illustration of the equalities of Eq. 55 in the disk $\mathcal{D}_{1 / 2}$.

Figure 6: Invariance of the Hilbert distance under observer changes: illuminants $C$ and $G$, and colors $H$ and $H^{\prime \prime}$, compared with illuminants $C$ and $A$, and colors $F$ and $F^{\prime}$.

These discussions show clearly that the Hilbert metric is compatible with the only psychovisual data that we have at disposal. Here we have reported only three cases, but other three configurations related to Fig. 6.1 can be studied and our computations showed that they give rise to the same conclusions. We have only treated the case when colors, e.g. $F$ and $F^{\prime}$, have chromatic vectors collinear to the new observer chromatic vector, e.g. $\mathbf{v}_{f}^{1}$ and $\mathbf{v}_{f^{\prime}}^{1}$ are collinear to $\mathbf{v}_{a}^{1}$ in this first situation. Dealing with arbitrary colors needs the introduction of more sophisticated mathematical tools to take into account the general addition law for non-collinear vectors. We prefer to not enter in such details in the present work and to postpone the general case for future research.

\section{Chromatic aberration, boost maps and the theoretical derivation of the outcome of Yilmaz's third experiment}

In this final section we show how eq. 21, the third and final result quoted by Yilmaz, can be obtained as a theoretical consequence of the trichromacy axiom. We will obtain this result by explaining how to recover the chromatic aberration effect from the trichromacy axiom, which happens to be related to how pure chromatic states generate Lorentz boost maps which act on the space of chromatic vectors.

\subsection{One parameter subgroups of boost maps}

Let us recall that the pure chromatic states are given by density matrices of the form:

$$
\rho(\mathbf{v})=\frac{1}{2}\left(I d_{2}+\mathbf{v} \cdot \sigma\right)=\frac{1}{2}\left(\begin{array}{cc}
1+v_{1} & v_{2} \\
v_{2} & 1-v_{1}
\end{array}\right),
$$

where $\mathbf{v}=\left(v_{1}, v_{2}\right)$ is a unit vector of $\mathbb{R}^{2}$. We have the following result.

Proposition 7.1 Every pure chromatic state generates a one-parameter subgroup of Lorentz boosts [3]. 
Proof. The matrix

$$
A\left(\mathbf{v}, \zeta_{0}\right)=\exp \left(\zeta \frac{\mathbf{v} \cdot \sigma}{2}\right)
$$

with $\zeta_{0}$ a real parameter, is an element of the group $P S L(2, \mathbb{R})$. Using the action of $P S L(2, \mathbb{R})$ on $\mathcal{H}(2, \mathbb{R})$ we can consider the matrices given by:

$$
\sigma_{i} \longmapsto A\left(\mathbf{v}, \zeta_{0}\right) \sigma_{i} A\left(\mathbf{v}, \zeta_{0}\right),
$$

for $i=0,1,2$ with $\sigma_{0}=I d_{2}$. The matrix with entries

$$
M\left(\mathbf{v}, \zeta_{0}\right)_{i j}=\frac{1}{2} \operatorname{Trace}\left(\sigma_{i} A\left(\mathbf{v}, \zeta_{0}\right) \sigma_{j} A\left(\mathbf{v}, \zeta_{0}\right)\right)
$$

is the matrix

$$
M(\zeta)=\left(\begin{array}{ccc}
\cosh \left(\zeta_{0}\right) & v_{1} \sinh \left(\zeta_{0}\right) & v_{2} \sinh \left(\zeta_{0}\right) \\
v_{1} \sinh \left(\zeta_{0}\right) & 1+v_{1}^{2}\left(\cosh \left(\zeta_{0}\right)-1\right) & v_{1} v_{2}\left(\cosh \left(\zeta_{0}\right)-1\right) \\
v_{2} \sinh \left(\zeta_{0}\right) & v_{1} v_{2}\left(\cosh \left(\zeta_{0}\right)-1\right) & 1+v_{2}^{2}\left(\cosh \left(\zeta_{0}\right)-1\right)
\end{array}\right),
$$

with $\zeta=\tanh \left(\zeta_{0}\right)\left(v_{1}, v_{2}\right)$.

For instance, if $\mathbf{v}=(1,0)$ then:

$$
M(\zeta)=\left(\begin{array}{ccc}
\cosh \left(\zeta_{0}\right) & \sinh \left(\zeta_{0}\right) & 0 \\
\sinh \left(\zeta_{0}\right) & \cosh \left(\zeta_{0}\right) & 0 \\
0 & 0 & 1
\end{array}\right)
$$

One can easily check that, in this case, the pure chromatic vector $(\cos \theta, \sin \theta) / 2$ is sent to the pure chromatic vector $\mathbf{w}=\left(w_{1}, w_{2}\right)$ with:

$$
\left\{\begin{aligned}
2 w_{1} & =\frac{\tanh \left(\zeta_{0}\right)+\cos \theta}{1+\tanh \left(\zeta_{0}\right) \cos \theta} \\
2 w_{2} & =\frac{\left(1-\tanh \left(\zeta_{0}\right)^{2}\right)^{1 / 2} \sin \theta}{1+\tanh \left(\zeta_{0}\right) \cos \theta} .
\end{aligned}\right.
$$

This example is precisely the one that describes mathematically the color effect brought to light by the third Yilmaz experiment.

\subsection{A theoretical derivation of the outcome of Yilmaz's third experi- ment}

The dynamics given by eq. 62 is nothing else than the dynamics of the relativistic aberration effect [14. It allows, as already suggested by Yilmaz, to explain the results of the third experiment described in 26.

The pure chromatic vector $(0,1) / 2$ is sent to the pure chromatic vector with coordinates $\left(\tanh \left(\zeta_{0}\right),\left(1-\tanh \left(\zeta_{0}\right)^{2}\right)^{1 / 2}\right) / 2$ whereas the pure chromatic vector $(1,0) / 2$ remains unchanged. When the rapidity $\zeta_{0}$ increases, $\tanh \left(\zeta_{0}\right)$ approaches 1 and the vector $\left(\tanh \left(\zeta_{0}\right),\left(1-\tanh \left(\zeta_{0}\right)^{2}\right)^{1 / 2}\right) / 2$ approaches the vector $(1,0) / 2$. At the limit $\tanh \left(\zeta_{0}\right)=1$, every pure chromatic vector $(\cos \theta, \sin \theta) / 2$ is sent to the vector $(1,0) / 2$, except the vector $(-1,0) / 2$.

This means that every pure chromatic vector, except the green pure chromatic vector, can be transformed to a pure chromatic vector arbitrarily close to the red pure chromatic vector under the Lorentz boost of eq. (61) if the rapidity $\zeta_{0}$ is sufficiently large.

Equation 62 allows us to provide a theoretical explanation of the results of Yilmaz's third experiment. To this aim, note that $w_{1}$ is the cosine of the angle of the ray from the achromatic vector to the image of the chromatic vector $(\cos \theta, \sin \theta) / 2$ viewed under the initial illuminant $I$, whereas

$$
\bar{w}_{1}=\frac{-\tanh \left(\zeta_{0}\right)+\cos \theta}{1-\tanh \left(\zeta_{0}\right) \cos \theta}
$$


is the cosine of the angle of the ray from the achromatic vector to the image of the chromatic vector $(\cos \theta, \sin \theta) / 2$ viewed under the illuminant $I^{\prime}$. As a consequence, under the illuminant $I^{\prime}$, the expected yellow chromatic vector given by $\theta=\pi / 2$ is in fact the greenish chromatic vector given by $\cos \theta=-\tanh \left(\zeta_{0}\right)$.

\section{Conclusion}

In this paper we have strengthen the novel quantum theory of color perception proposed in [3] and we extend it to incorporate also relativistic phenomena, resulting in a coherent relativistic quantum theory of color perception.

We have shown that the noticeable, yet heuristic, intuition of Yilmaz 26] regarding the relativistic nature of color perception can be incorporated in a rigorous mathematical setting that can be built from the single axiom of trichromacy.

We have obtained this result by following the hint given by Mermin's alternative, and perhaps more profound, reconstruction of the special theory of relativity from Einstein-Poincaré addition law for velocity vectors. This led us to define and analyze the crucial concept of perceptual chromatic vector and to show that such vectors actually satisfy Einstein-Poincaré addition law.

Quite surprisingly, this fact also allowed us to coherently endow the space of perceptual chromatic vectors with the Hilbert metric, which we verify to be in accordance with known experimental results, thus underline the importance of such a distance in color perception.

We consider fascinating that both the relativistic and the quantum components of the theory of color perception that we describe in this paper are based on unconventional and quite rarely used approaches: Mermin's viewpoint on special relativity and Jordan's algebraic perspective on quantum theories.

We deem that a significant part of our contribution is also represented by a whole new nomenclature that we introduced in sections 24.2 and 5.1 . This is an unavoidable step when building, or refining, a novel theory. Nevertheless, in doing so we have tried to remain as close as possible to terms already present in quantum mechanics, special relativity and standard colorimetry, respectively.

We are currently investigating the mathematical details of the extension of our proposal from collinear chromatic vectors to the general case in which chromatic vectors do not necessarily lie on the same axis. Moreover, we are also analyzing the possibility to explain well-known perceptual effects, see e.g. [8] and [1], with the theoretical framework discussed here.

We also consider interesting to study how the novel objects and formalism that we have introduced in this work can be used for practical colorimetric purposes and how they relate to existing color spaces represented in cylindrical coordinates such as the HSV space. To this aim, and also to more finely test our proposal, it is paramount to complement the exiguous psychovisual data that we currently have at disposal and possibly to design new kind of experiments.

\section{Appendix - Description of Yilmaz experiments}

The generic apparatus for the experiments is shown in Fig. 7. where we can see two identical rooms $R_{1}$ and $R_{2}$, separated by a common wall with a thin hole and illuminated by the sources of light $S_{1}$ and $S_{2}$. Both rooms are painted with a non-selective Lambertian white paint. A piece of white paper is divided in two parts and they are placed in the rooms, so that an observer can perceive them simultaneously. The key point is that one piece is seen directly and the other through the hole.

The illumination $S_{1}$ of room $R_{1}$ will always be provided by near-daylight broadband illuminants. Instead, the illumination of room $R_{2}$ will be provided by a light source $S_{2}$ that can also be narrow-band. The perceived colors are compared with the help of a set of Munsell chips enlighted by the same illuminant under which the observer is adapted. 

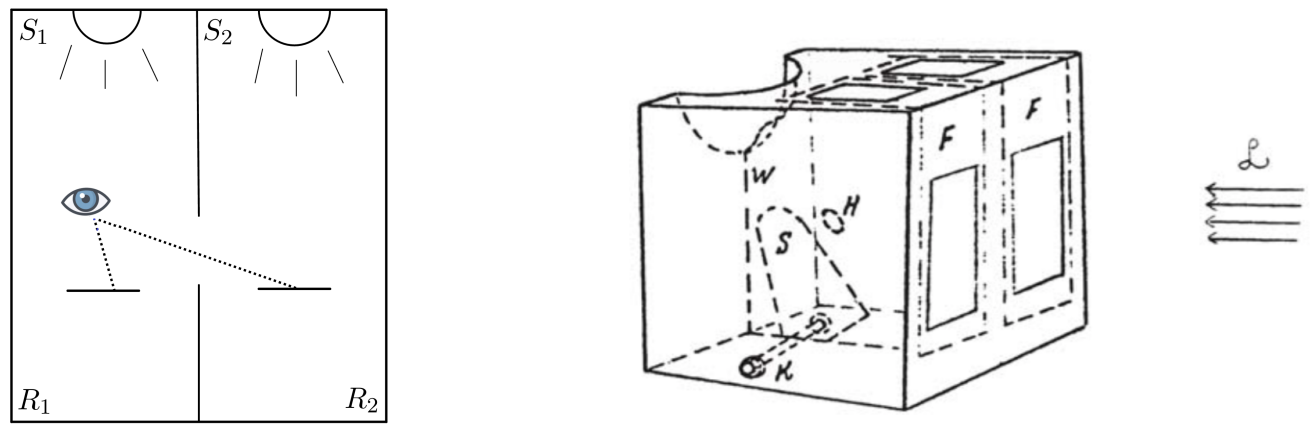

Figure 7: The experimental apparatus considered by Yilmaz. The image on the right is from Inter-Society Color Council News, Issue 419, Jan/Feb 2006, by kind concession of M. H. Brill, whom we would like to thank for sharing this reference with us.

A detailed analysis of the interpretation and feasibility of the experiments is available in [18, here we quote directly Yilmaz [26] to allow the reader to make up her or his own mind about them.

\subsection{The first experiment}

'If the sources $S_{1}$ and $S_{2}$ are chosen to be two different illuminants of near-daylight chromaticity, $I$ and $I^{\prime}$, then the wall of each room is perceived as white by the observer in the room but the wall of the other room, as seen through the hole, appears chromatically colored. Furthermore, if $R_{2}$ appears with the saturation $\sigma$ from $R_{1}$, then $R_{1}$ appears with the saturation approximately $-\sigma$ from $R_{2}$, the minus sign indicating that the hue is complementary to the former hue.'

\subsection{The second experiment}

'If $S_{2}$ is chosen to be a single-frequency source, say, that corresponding to the long-wave (red) extreme of the visible spectrum $\bar{R}$, then the saturation $\Sigma$ observed through the hole (observer being in $R_{1}$ ) is too high to be duplicated by any of the Munsell chips, and remains practically the same if we change the illuminant from $I$ to $I^{\prime}$ in $R_{1}$.'

\subsection{The third experiment}

'Let $S_{2}$ be a source of frequency corresponding to the yellow part of the spectrum, $\bar{Y}$, separated in the hue circle by 90 degrees from spectrum red, $\bar{R}$. Then if we change the illuminant in $R_{1}$ from $I$ to $I^{\prime}$, the hue of $\bar{Y}$ is seen to change by an amount $\varphi$ such that $\sin \varphi \simeq \sigma / \Sigma$. No variation seems to take place in its saturation.'

\section{References}

[1] A. Ashtekar, A. Corichi, and M. Pierri. Geometry in color perception. Black Holes, Gravitational Radiation and the Universe, pages 535-550, 1999.

[2] John C Baez. Division algebras and quantum theory. Foundations of Physics, 42(7):819-855, 2012 .

[3] M. Berthier. Geometry of color perception. Part 2: perceived colors from real quantum states and Hering's rebit. The Journal of Mathematical Neuroscience, 10(1):1-25, 2020. 
[4] RW Burnham, RM Evans, and SM Newhall. Prediction of color appearance with different adaptation illuminations. JOSA, 47(1):35-42, 1957.

[5] Bruno Colbois and Constantin Vernicos. Les géométries de Hilbert sont à géométrie locale bornée. In Annales de l'institut Fourier, volume 57, pages 1359-1375, 2007.

[6] C. P. Crocetti and W. P. Bethke. Scientific and Technical Aerospace Reports, 1.

[7] Gerard G Emch. Algebraic methods in statistical mechanics and quantum field theory. Courier Corporation, 2009.

[8] M.D. Fairchild. Color appearance models. Wiley, 2013.

[9] Vladimir Fock. The theory of space, time and gravitation. Elsevier, 2015.

[10] Alan Gilchrist, Christos Kossyfidis, Frederick Bonato, Tiziano Agostini, Joseph Cataliotti, Xiaojun Li, Branka Spehar, Vidal Annan, and Elias Economou. An anchoring theory of lightness perception. Psychological review, 106(4):795, 1999.

[11] G. Gronchi and E. Provenzi. A variational model for context-driven effects in perception and cognition. Journal of Mathematical Psychology, 77:124-141, 2017.

[12] Pascual Jordan. Über eine klasse nichtassoziativer hyperkomplexer algebren. Nachrichten von der Gesellschaft der Wissenschaften zu Göttingen, Mathematisch-Physikalische Klasse, 1932:569-575, 1932.

[13] Frederick AA Kingdom. Lightness, brightness and transparency: A quarter century of new ideas, captivating demonstrations and unrelenting controversy. Vision research, 51(7):652673, 2011.

[14] Lev Davidovich Landau and Evgenii Mikhailovich Lifshitz. The classical theory of fields. Pergamon, 1971.

[15] Kurt Lechner. Classical Electrodynamics. Springer, 2018.

[16] N David Mermin. Relativity without light. American Journal of Physics, 52(2):119-124, 1984.

[17] V. Moretti. Spectral theory and quantum mechanics: mathematical foundations of quantum theories, symmetries and introduction to the algebraic formulation. Springer, 2017.

[18] N. Prencipe, V. Garcin, and E. Provenzi. Origins of hyperbolicity in color perception. Journal of Imaging, 42(6):1-19, 2020.

[19] Edoardo Provenzi. Geometry of color perception. Part 1: Structures and metrics of a homogeneous color space. The Journal of Mathematical Neuroscience, 10(1):1-19, 2020.

[20] H.L. Resnikoff. Differential geometry and color perception. Journal of Mathematical Biology, 1:97-131, 1974 .

[21] Walter Rudin. Real and complex analysis. Tata McGraw-hill education, 2006.

[22] B. Russell. The problems of philosophy. OUP Oxford, 2001.

[23] Steven K Shevell and Paul R Martin. Color opponency: tutorial. JOSA A, 34(7):1099-1108, 2017.

[24] Franco Strocchi. An introduction to the mathematical structure of quantum mechanics: a short course for mathematicians, volume 28. World Scientific, 2008.

[25] G. Wyszecky and W. S. Stiles. Color science: Concepts and methods, quantitative data and formulas. John Wiley \& Sons. John Wiley \& Sons, 1982.

[26] H. Yilmaz. On color perception. Bulletin of Mathematical Biophysics, 24:5-29, 1962. 